Background Patients express a preference to die at home. This has influenced National Policies, with the focus in health care to support as many patients to achieve this as possible. Annual deaths are expected to rise, but less than one in ten people achieving home deaths before $2030 .^{3}$ North Wales has a wide geographical area with services to support palliative patients varying across the different areas. This research will enable effective methods of support and types of interventions in North Wales to be identified; and thus be a starting point towards increasing the numbers of patients able to die at home. The results will be helpful to other areas nationally and internationally.

Aims To understand the experience of caring for dying patients at their own home in North Wales, and clarify which aspects enabled a 'good death' at home for patients who expressed a wish to die in their own home. To identify which support services were involved and to clarify the interventions that enabled or hindered a patient to remain at home to die.

Methods A multi perspective qualitative study of semi structured interviews of 18 bereaved carers and 18 Health Care Professionals involved in patients' care to die at home. All interviews were recorded and transcribed.

Results Thematic analysis of information has taken place and the benefits and challenges of caring for dying patients in their own home have been explored.

Conclusion Recommendations regarding views on effective support mechanisms and interventions will be highlighted and provide guidance on supporting patients to die in their preferred place of home.

\section{P 008A CARER AND HEALTH CARE PROFESSIONALS' VIEWS ON CARING FOR DYING PATIENTS IN THEIR HOMES IN NORTH WALES}

Jacqueline Pottle, ${ }^{1,2}$ Richard Neal, ${ }^{1}$ Marlise Poolman, ${ }^{1,2}$ Julia Hiscock'. 'Wrexham Academic unit, Bangor University, North Wales, Great Britain; ${ }^{2}$ Betsi Cadwladr University Health Board, North Wales, Great Britain 